\section{Psychiatric Symptoms in Transsexualism}

SIR: Before we draw the obvious conclusion from the paper of Mate-Kole et al (Journal, April 1988, $152,550-553)$ that treatment of transsexualism by adopting the preferred gender role and later surgery reduces their psychological symptoms, we need to know the characteristics and numbers of those who drop out between the stages of treatment described in the paper. It is possible, but unlikely, that there are a few highly psychiatrically disturbed individuals who present for assessment but drop out before they are accepted for the waiting list or before surgery takes place. This would alter the means and standard deviations of the scores of the groups in the directions of improving the scores who continue in treatment. Such an effect would be ruled out with the prospective study mentioned by the authors, but in the meantime it would be nice to be reassured on this point.

St George's Hospital Medical School

JOHN M. KELLETT

Cranmer Terrace

London SW17 ORE

SIR: The study as indicated was retrospective, and at the time of examination there were no drop-outs in the two pre-operative groups. Table 1 of our paper elaborates on the psychiatric background of these patients.

It is not uncommon in our daily clinic practice for patients to drop out between treatment stages, bearing in mind that a sizeable proportion of patients who present in gender identity clinics display other gender identity disorders and psychiatric disturbance, and therefore do not fit the criteria for diagnosis of transsexualism (Mate-Kole \& Freschi, 1988). Certainly, in our clinic less than $20 \%$ of the patients ever reach the operation stage (Mate-Kole et al, 1987), this being the reason that we suggested rigorous prospective studies be conducted.

Camp Hill Hospital

Charles Mate-Kole

1763 Robie Street

Halifax

Nova Scotia

Canada B3H $3 G 2$

\section{References}

MATE-Kole, C. \& Freshi, M. (1988) Psychiatric aspects of sex reassignment surgery. British Journal of Hospital Medicine, 153-158.

Mate-Kole, C., Montgomery, D., Dalrymple, J. \& Hirsch, S. (1987) Gender reassignment today. British Medical Journal, 295, 997.

\section{Risk Factors in Schizophrenia}

SIR: We were interested to read Baron \& Gruen's study (Journal, April 1988, 152, 460-465) showing an increased risk for schizophrenia and 'spectrum' disorders among first-degree relatives of schizophrenic patients born in the winter or spring. We have recently studied the relationship between season of birth and a number of variables, including family history, in a sample of 126 patients who fulfilled the RDC for schizophrenia or schizoaffective disorder, schizophrenic type. Patients were judged to have a positive family history if a first-degree relative had suffered from schizophrenia, schizoaffective disorder, manic depressive psychosis (as defined in ICD-9), or unspecified psychosis.

A positive family history was found in 25 out of 67 (37\%) patients born in the winter or spring (December-May), compared with 12 out of $59(20 \%)$ born in the summer or autumn (June-November). This difference just failed to attain statistical significance at the $5 \%$ level $(Z=1.88, P=0.06$, Fisher's exact test, 2-tailed).

Drs Baron \& Gruen actually failed to find differences between family history positive and negative cases. In their study a relationship between familial load and season of birth was only seen when morbid risk data were analysed. As they, and others, have pointed out, classifying patients into familial and nonfamilial cases has a number of limitations, all of which reduce the likelihood of demonstrating relationships between familial load and other variables. It seems likely that our modest success in demonstrating a relationship between family history and season of birth was due to the fact that our sample $(n=126)$ was larger than Drs Baron \& Gruen's $(n=88)$.

Michael J. OWEN SHÔN W. LEWIS

Genetics Section

Institute of Psychiatry

London SE5 8 AF

\section{Therapy-Resistant Depression}

SIR: Leonard (Journal, April 1988, 152, 453-459) argues that lithium enhances presynaptic serotonergic transmission in animals and that its effect in man is related to its antidepressant effect by synergism with serotonergic postsynaptic enhancing drugs. Part of his argument is based on the assertion that lithium is "a poor antidepressant when used alone". I do not want to rehash arguments I have already made about the interpretation of the literature (Worrall, 1986) but I am surprised at the two references he quotes to support his assertion. Mendels 